\title{
Preliminary Results from a pilot study - Intensive Case Management to prevent relapse among persons with Alcohol Withdrawal Delirium Tremens
}

\author{
Julian A. Joseph Arthur ${ }^{1}$, R. Dhanasekara Pandian², Pratima Murthy ${ }^{3}$ \\ ${ }^{1}$ Ph.D Scholar, Department of Psychiatric Social Work, National Institute of Mental Health and Neurosciences \\ (NIMHANS), Bangalore. \\ ${ }^{2}$ Additional Professor, Department of Psychiatric Social Work, National Institute of Mental Health and Neurosciences \\ (NIMHANS), Bangalore. \\ ${ }^{3}$ Professor of Psychiatry, Centre for Addiction Medicine, Department of Psychiatry, National Institute of Mental \\ Health and Neurosciences (NIMHANS), Bangalore. \\ E-mail - arthurjoseph7@gmail.com
}

\begin{abstract}
Introduction: Alcohol withdrawal Delirium Tremens is a serious medical emergency. If not addressed, it could lead to serious complications including death. Given its manifestations and frequent accompaniment by seizures, interventions have been pharmacological in nature. Relapse prevention studies addressing this population are very sparse. In the current study, we report the results of a pilot study on Intensive Case Management for relapse prevention in persons with Alcohol Withdrawal Delirium.

Methodology: A total of 10 patients were selected using a consecutive sampling technique. 5 patients received Intensive Case Management as an adjunct to routine treatment while the other 5 cases received treatment as usual (TAU). The patients were assessed at baseline, at 1 month post intervention and at 3 months post-intervention respectively. Outcome measures such as addiction severity, motivation, self efficacy and quality of life were assessed at different time points.

Results: The ICM group showed better outcomes than the TAU group. 4 patients in the ICM group were abstinent while compared to 1 in the TAU group at the end of the $3^{\text {rd }}$ month follow up. All the patients in the ICM group were engaged in the treatment and showed better follow rates as compared to the TAU group where at the end of the study period 3 patients were lost to follow up. The ICM group showed better outcomes on readiness to change, self efficacy and quality of life at the end of 3 months.

Conclusion: Our findings suggest that patients who received Intensive Case Management were engaged in treatment with higher retention rates and better outcomes. The results of the pilot study suggest that ICM is feasible to deliver after pharmacological treatment and may have some beneficial outcomes. Further systematic studies are warranted to test this intervention with larger groups and for long-term effects.
\end{abstract}

Key Words: Alcohol Dependence Syndrome, Delirium Tremens, Feasibility, Intensive Case Management.

(Paper received $-21^{\text {st }}$ December 2016, Peer review completed $-4^{\text {th }}$ January 2017 , Accepted $-6^{\text {th }}$ January 2017)

\section{INTRODUCTION}

Alcohol Use Disorders (AUDs) are a major cause of physical and mental health problems [1] for which an array of psycho-social interventions have been shown to be efficacious [2-3]. Alcohol withdrawal delirium (Delirium Tremens, DT), is a severe complication which may be preceded or complicated by seizures [4]. Literature on Alcohol Withdrawal Delirium (AWD) in India is scarce as highlighted in a review and these groups of patients are not given importance in teaching programmes, research protocols and therapeutic 
strategies [5]. Being a medical emergency, pharmacological management is carried out [6]. However, literature on longer-term non-pharmacological interventions is lacking [7]. Patients with AWD that present to emergency rooms or psychiatric services mainly receive pharmacological treatment to recover from the episode of DT following which majority of them seek discharge without receiving adequate psycho-social interventions.

Pharmacological treatment is just the first step towards abstinence and extends into enrolling patients in long term treatment programmes [8], but this is not so in the case of patients with AWD due to their present state; early discharge and lack of insight are few of the reasons as to why this group of patients are found difficult to engage in treatment thereby resulting in frequent relapses and admissions. Apart from psycho-social issues that are common to persons with substance use, this group of patients are vulnerable to problems such as an irreversible damage to their brain, delay in the recovery process, impairment of attention and or executive functions leading to a further relapse of substance use, co-occurring medical complications and in certain cases death [9-10].

AUDs are chronic and relapsing in nature [11] patients with AWD would benefit from a more intensive approach than the routine clinical treatment such as the Intensive Case Management (ICM). ICM is an extension of the Assertive Community Treatment model by Stein and Test [12]. Studies on ICM in developed countries report it to be effective for persons with severe mental illness and co-morbid substance use disorders [13-14]. It is a chronic disease management strategy that employs active intervention to facilitate acute drug abuse treatment, provides long-term strategies for management including relapse monitoring and addresses psychosocial issues [15-16]. ICM remains one of the underutilized psychosocial interventions for chronic alcohol dependence. Here we report the results of the pilot study that tested the use of ICM for patients with AWD who sought treatment in a tertiary care hospital in India.

\section{METHODOLOGY}

The present study is a pilot study that was carried out at the Centre for Addiction Medicine, NIMHANS, Bengaluru, Karnataka, India. Ten (10) male patients aged $\geq 18$ years to 50 years; diagnosed with ADS as per the International Classification of Diseases (ICD-10), presenting with AWD, able to converse in either English or Kannada; and not using any other substance (except nicotine) were included using consecutive sampling. Persons with co-morbid or unstable medical or neurological conditions were excluded. Five patients received the psycho-social interventions using the Intensive Case Management and the other five patients received Treatment as Usual. The ethical approval was obtained from the institutional Human Ethics Committee of National Institute of Mental Health and Neuro Sciences (NIMHANS). The patients were explained about the study and written informed consent was obtained.

Treatment as Usual Group (TAU): The patients in the TAU group had received regular clinical services which included pharmacological and psycho-social interventions such as motivational interviewing and relapse prevention strategies including individual and group work sessions. But due to the early discharge these patients do not get all of the required services.

Table1 - Details of ICM

\begin{tabular}{|llll|}
\hline Details of Intensive Case Management & Baseline & T1 & T3 \\
\hline Home visits done by the researcher & 4 & 3 & 2 \\
\hline Telephonic contacts made by the researcher & 2 & 4 & 6 \\
\hline Follow ups done by the researcher & 1 & 2 & 3 \\
\hline T1- $1^{\text {st }}$ month follow up, T3 $-3^{\text {rd }}$ month follow up & & \\
\hline
\end{tabular}


Intervention group: The patients in the Intervention group received treatment as usual along with ICM which comprised of need based interventions focusing on managing craving and relapse, addressing family problems, information about the illness and tasks enhancing attention and concentration. Interventions were provided in the patient's home along with routine follow ups and telephonic contacts during the study period. Telephonic contacts were made to check the status of the patient as well as provide help during crisis. The patients were engaged in treatment and asked to come for follow up to review their progress regularly.

Tools for the study: The ICM group received the intervention after the base line assessments were completed. Both the groups were assessed on the following outcomes measures.

1. Addiction Severity Index (ASI) [17]: It is a semi-structured instrument used in face-to-face interviews that covers: medical, employment, drug and alcohol use, legal, family/social, and psychiatric components. The ASI obtains information about problem behaviours within the previous 30 days. It has excellent inter-rater reliabilities ranging from 0.84-0.95 with content, criterion and constructs validities and demonstrates good specificity and sensitivity. The scoring ranges from 0 to 1 , where a score of 0 indicates no or less severity and a higher score of 1 indicates a higher level of severity in the case of the domains on medical, alcohol and family except in the case of employment lower scores indicate a higher severity.

2. University of Rhode Island Change Assessment (URICA) [18]: is a 32 item self report measure that includes 4 subscales to assess motivational readiness to change (Pre-contemplation, Contemplation, Action and Maintenance). Response are given on a 5 point likert scale ranging from 1 (strong disagreement) to 5 (strong agreement). The psychometric property reveals that it has construct, content and criterion validity and reliability on internal consistency (Cronbachs alpha: 0.84 ). A score of 8 or lower depicts the stage of pre contemplation; 8-11 means that they are in the stage of contemplation; 11-14 says that they are in the stage of preparation and a score of 14 and above reveals that they are in the stage of maintenance.

3. Alcohol Abstinence Self Efficacy Scale (AASE) [19]: It assesses Bandura's construct of self efficacy and evaluates an individual's efficacy to abstain from drinking in over 20 situations that represent typical drinking cues. These situations from four sub scales which are negative affect, social or positive, physical and other concerns and cravings and urges. The psychometric property reveals that it has good construct validity and reliability internal consistency (Cronbachs alpha: 0.92). Scores ranging below 3.5 was rated as low self efficacy and above 3.5 indicated higher rate.

4. World Health Organization Quality of Life (WHOQOL BREF) [20] : It comprises of 26 items which provides a measure of an individual's perception to quality of life on 4 domains: physical health, psychological health, social relationships and environment. It has an internal consistency of Cronbachs alpha ranging from 0.64-0.84 with a test-retest reliability ranging between 0.66-0.87 and known to have good discriminant validity. Higher scores indicated higher quality of life.

\section{STATISTICAL ANALYSIS}

$\mathrm{R}$ Software (version 3.1.2) was used for statistical analysis. The socio-demographic and clinical related data was presented with frequencies, percentiles, mean and median. Since the sample size was a very small number to allow for a definitive analysis we used mean and standard deviation scores to compare the differences among persons with AWD on Addiction severity - alcohol; motivation, self efficacy and quality of life at baseline and at 1 month and 3 months following completion of the intervention.

\section{RESULTS}

All the patients who participated in the pilot study were Hindus by religion, living in nuclear families and married. The detailed socio demographic and clinical profiles of the patients from both the groups are presented in Table 2. 
Table 2 - Socio-demographic and clinical profile of patients with AWD

\begin{tabular}{|c|c|c|c|c|c|c|}
\hline \multirow[t]{2}{*}{ Profile } & & \multicolumn{2}{|l|}{ ICM } & \multicolumn{3}{|c|}{ TAU } \\
\hline & & \multirow{2}{*}{$\frac{\text { Mean } \pm \text { SD }}{37.60 \pm 6.19}$} & Median & \multicolumn{2}{|c|}{ Mean \pm SD } & Median \\
\hline \multicolumn{2}{|l|}{ Age } & & 40.00 & \multicolumn{2}{|c|}{$37.20 \pm 6.87$} & 35.00 \\
\hline \multicolumn{2}{|l|}{ Years of Education } & $6.20 \pm 4.66$ & 5.00 & \multicolumn{2}{|c|}{$7.20 \pm 2.28$} & 7.00 \\
\hline \multicolumn{2}{|l|}{ Individual Income (INR) } & $16200 \pm 8075$ & 15000 & \multicolumn{2}{|c|}{$10800 \pm 6905$} & 10000 \\
\hline \multicolumn{2}{|c|}{ Age of onset of dependence } & $24.20 \pm 3.49$ & 26.00 & \multicolumn{2}{|c|}{$25.00 \pm 1.732$} & 26.00 \\
\hline \multicolumn{2}{|c|}{ Duration of Dependence (years) } & $14.20 \pm 4.08$ & 15.00 & \multicolumn{2}{|c|}{$13.00 \pm 6.782$} & 14.00 \\
\hline \multicolumn{2}{|c|}{ Abstinence from alcohol(months) } & $34.00 \pm 18.16$ & 30.00 & \multirow{2}{*}{\multicolumn{2}{|c|}{$\frac{45.00 \pm 25.981}{280 \pm 130.38}$}} & 30.00 \\
\hline \multicolumn{2}{|c|}{ Money spent on alcohol (per day) } & $320.00 \pm 109.54$ & 300.00 & & & 200.00 \\
\hline \multicolumn{3}{|c|}{ (2) } & & \%) & $\mathbf{N}$ & (\%) \\
\hline \multirow{3}{*}{$\begin{array}{l}\text { Educational } \\
\text { qualification }\end{array}$} & Illiterat & & & & 0 & - \\
\hline & Primar & & & & 2 & $40 \%$ \\
\hline & High sc & & & & 3 & $60 \%$ \\
\hline & Unemp & & & & 1 & $20 \%$ \\
\hline Occupation & Unskill & rker & & & 2 & $40 \%$ \\
\hline & Semi-sk & worker & & & 2 & $40 \%$ \\
\hline Socio economic status & Middle & r middle & & & 1 & $20 \%$ \\
\hline & Lower/ & middle & & & 2 & $40 \%$ \\
\hline & Lower & & & & 2 & $40 \%$ \\
\hline
\end{tabular}

Patients receiving ICM showed better outcomes at $3^{\text {rd }}$ month follow up than the TAU group. This was also evident by the number of patients that relapsed at 1 month and 3 months follow up respectively was lesser in the ICM group than the TAU group.

Table 3 - Difference in outcome scores across the time points between the two groups

\begin{tabular}{|c|c|c|c|c|c|c|c|c|c|c|c|c|}
\hline & \multicolumn{6}{|c|}{ ICM $(\mathrm{N}=5)$} & \multicolumn{6}{|c|}{ TAU $(\mathbf{N}=5)$} \\
\hline & \multicolumn{2}{|c|}{ BASELINE } & \multicolumn{2}{|l|}{$\mathrm{T} 1$} & \multicolumn{2}{|l|}{ T3 } & \multicolumn{2}{|c|}{ BASELINE } & \multicolumn{2}{|l|}{ T1 } & \multicolumn{2}{|l|}{ T3 } \\
\hline STATUS & $\mathbf{A B}$ & $\mathbf{R E}$ & $\overline{\mathrm{AB}}$ & RE & $\overline{A B}$ & RE & AB & $\overline{R E}$ & $\overline{A B}$ & RE & $\overline{A B}$ & RE \\
\hline \multirow[t]{2}{*}{$\mathrm{N}$} & 0 & 5 & 5 & 0 & 4 & 1 & 0 & 5 & 3 & 2 & 1 & 4 \\
\hline & \multicolumn{2}{|c|}{ Mean \pm SD } & \multicolumn{2}{|c|}{ Mean \pm SD } & \multicolumn{2}{|c|}{ Mean \pm SD } & \multicolumn{2}{|c|}{ Mean \pm SD } & \multicolumn{2}{|c|}{ Mean \pm SD } & \multicolumn{2}{|c|}{ Mean \pm SD } \\
\hline Motivation & \multicolumn{2}{|c|}{$8.20 \pm 0.51$} & \multicolumn{2}{|c|}{$13.67 \pm 0.15$} & \multicolumn{2}{|c|}{$12.80 \pm 0.45$} & \multicolumn{2}{|c|}{$7.97 \pm 0.63$} & \multicolumn{2}{|c|}{$13.20 \pm 1.09$} & \multicolumn{2}{|c|}{$10.20 \pm 0.45$} \\
\hline Self efficacy & \multicolumn{2}{|c|}{$2.02 \pm 0.16$} & \multicolumn{2}{|c|}{$4.00 \pm 0.05$} & \multicolumn{2}{|c|}{$3.17 \pm 0.77$} & \multicolumn{2}{|c|}{$1.96 \pm 0.25$} & \multicolumn{2}{|c|}{$3.31 \pm 0.75$} & \multicolumn{2}{|c|}{$2.50 \pm 0.78$} \\
\hline $\begin{array}{l}\text { Quality of } \\
\text { life }\end{array}$ & \multicolumn{2}{|c|}{$36.22 \pm 6.09$} & \multicolumn{2}{|c|}{$64.73 \pm 12.02$} & \multicolumn{2}{|c|}{$61.36 \pm 17.16$} & \multicolumn{2}{|c|}{$36.48 \pm 5.22$} & \multicolumn{2}{|c|}{$58.72 \pm 9.40$} & \multicolumn{2}{|c|}{$45.39 \pm 10.43$} \\
\hline
\end{tabular}

The mean difference of the outcome scores across different time points are depicted in table 3 . Though at the end of the third month follow up there is decline in the scores when compared to the first month follow up, the results highlight that the ICM group was better than the TAU group of patients. The mean outcome scores in the ICM group at the end of the 3 months showed that they had better scores on motivation (12.80 \pm 0.45$)$; self efficacy $(3.17 \pm 0.77)$ and better quality of life $(61.36 \pm 17.16)$ as compared to the TAU group. 
Figure 1 - Motivation scores between the two groups across the three time points

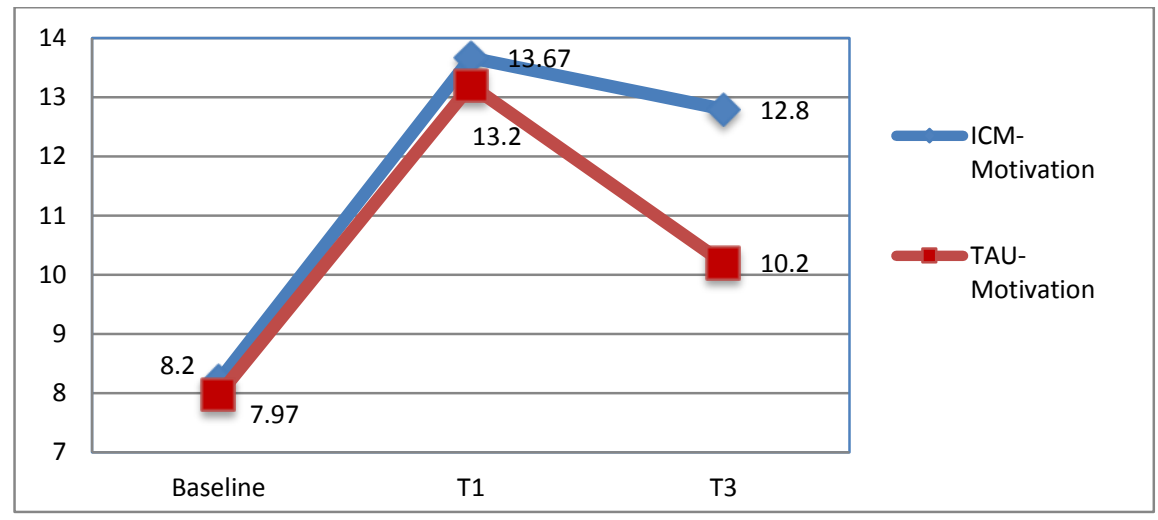

Figure 2 - Self Efficacy scores between the two groups across the three time points

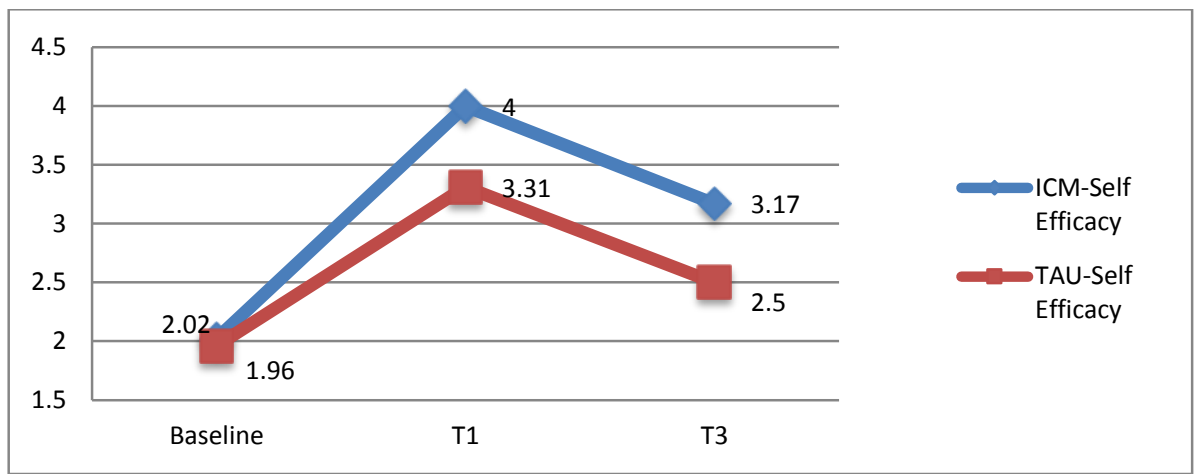

Figure 3 - Quality of Life between the two groups across the three time points

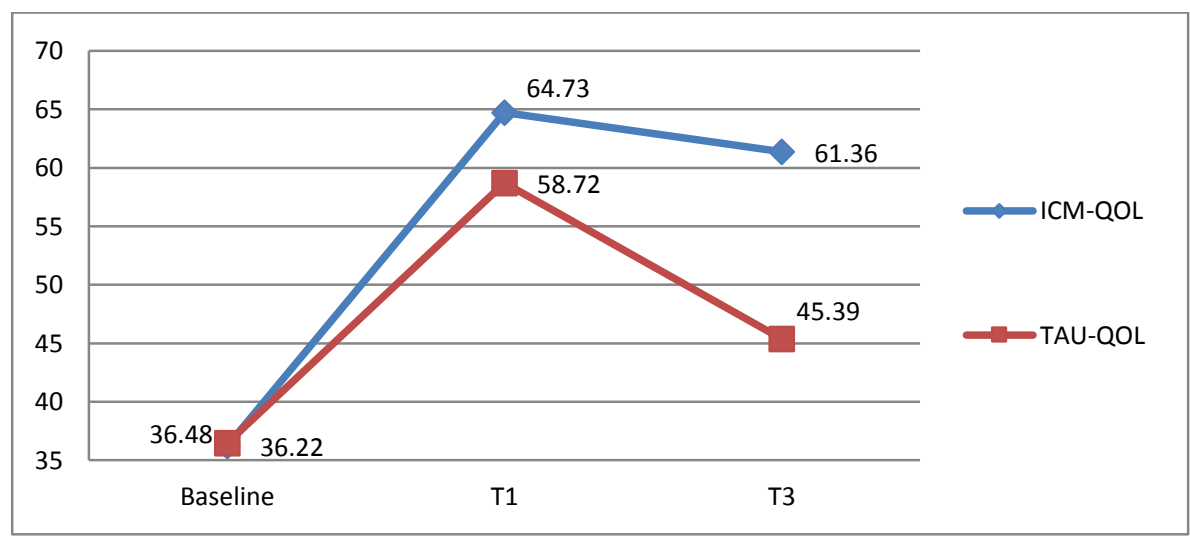

\section{DISCUSSION}

The results highlight the beneficial outcomes of patients who received psycho-social intervention using ICM. Firstly the ICM group showed better abstinence rates and few relapses at the end of 3 months than those in the TAU group. Patients admitted in the centre and are advised to undergo a 3 week programme that incorporates specific psycho-social interventions and family interventions as well. However patients with AWD take a longer period to recover before any interventions can be initiated. Lack of insight and early discharge from the hospital are reasons as to why psycho-social interventions are usually not initiated. Owing to which the crux of interventions that are to be delivered during the recovery phase are 
not provided resulting in frequent relapse and re-admission as seen in the TAU group during the follow up periods. On the other hand, active engagement through home visits, regular telephonic contacts and routine follow ups which are the characteristics of ICM, along with providing motivational interviewing and teaching patients relapse prevention strategies could have helped the patients in the ICM group. Indicating that ICM might be beneficial in increasing the number of abstinent days and engaging patients in treatment programme which is similar to that of results [14-15, 21-22].

To conclude the results of the current pilot study emphasizes on the need for developing the use of ICM and that preliminary findings suggest that this may be beneficial. Considering that this group of patients are very vulnerable and require an intensive treatment approach, ICM was found favorable in achieving abstinence, improved engagement in treatment and succeeded in preventing drop out [12-15]. We can conclude from this pilot study that delivering psycho-social interventions using the Intensive Case Management approach to patients AWD is both desirable and feasible. This area is grossly under researched and further systematic studies are warranted to test this intervention with larger groups and for long-term effects of ICM for persons with severe forms of AWD.

\section{REFERENCES}

1. Benegal V. India: alcohol and public health. Addiction 2005;100(8):1051-6.

2. Xiang X. A Review of Interventions for Substance Use Among Homeless Youth. Res Soc Work Pract 2013; 23(1):34-45.

3. Klimas J, Field C-A, Cullen W, O'Gorman C, Glynn L, Keenan E, et al. Psychosocial interventions to reduce alcohol consumption in concurrent problem alcohol and illicit drug users: Cochrane Review. Systematic Reviews 2013;2(1):3.

4. WHO. The ICD-10 Classification of Mental and Behavioural Disorders: diagnostic criteria for research. Geneva: World Health Organization, 1993.

5. Pinto C. Indian research on acute organic brain syndrome: Delirium. Indian J Psychiatry 2010;52(Supp1 1):S139-47.

6. Kattimani S, Bharadwaj B. Clinical management of alcohol withdrawal: A systematic review. Industr Psychiatry J 2013;22(2):100-8.

7. Mayo-Smith MF, Beecher LH, Fischer TL, Gorelick DA, Guillaume JL, Hill A, Jara G, Kasser C, Melbourne J. Management of alcohol withdrawal delirium: an evidence-based practice guideline. Arch Internal Med 2004;164(13):1405-12..

8. Blondell RD. Ambulatory detoxification of patients with alcohol dependence. Am Fam Physician 2005;71(3):495-502.

9. Borlikova GG, Elbers NA, Stephens DN. Repeated withdrawal from ethanol spares contextual fear conditioning and spatial learning but impairs negative patterning and induces over-responding: evidence for effect on frontal cortical but not hippocampal function? Eur J Neurosci 2006;24(1):205-16.

10. Loeber S, Duka T, Márquez HW, Nakovics H, Heinz A, Mann K, Flor H. Effects of repeated withdrawal from alcohol on recovery of cognitive impairment under abstinence and rate of relapse. Alcohol Alcohol 2010;45(6):541-7.

11. McLellan AT. Have we evaluated addiction treatment correctly? Implications from a chronic care perspective. Addiction 2002;97(3):249-52.

12. Stein LI, Test M. Alternative to mental hospital treatment: I. conceptual model, treatment program, and clinical evaluation. Arch Gen Psychiatry 1980;37(4):392-7.

13. Burns T, Catty J, Dash M, Roberts C, Lockwood A, Marshall M. Use of intensive case management to reduce time in hospital in people with severe mental illness: systematic review and meta-regression. BMJ 2007;335(7615):336.

14. Dieterich M, Irving CB, Park B, Marshall M. Intensive case management for severe mental illness. Cochr Database Syst Rev 2010.

15. Morgenstern J, Blanchard KA, McCrady BS, McVeigh KH, Morgan TJ, Pandina RJ. Effectiveness of intensive case management for substance-dependent women receiving temporary assistance for needy families. Am J Pub Health 2006;96(11):2016-23.

16. Morgenstern J, Blanchard KA, Kahler C, Barbosa KM, McCrady BS, McVeigh KH. Testing mechanisms of action for intensive case management. Addiction 2008;103(3):469-77. 
17. McLellan AT, Luborsky L, Woody GE, O'Brien CP. An improved diagnostic evaluation instrument for substance abuse patients. The Addiction Severity Index. J Nerv Mental Disord 1980;168(1):26-33.

18. McConnaughy EA, DiClemente CC, Prochaska JO, Velicer WF. Stages of change in psychotherapy: A follow-up report. Psychotherapy 1989;26(4):494-503.

19. DiClemente CC, Carbonari JP, Montgomery RP, Hughes SO. The Alcohol Abstinence Self-Efficacy scale. J Stud Alcohol 1994;55(2):141-8.

20. WHO Group. Development of the World Health Organisation WHOQOL - BREF Quality of Life assessment. Psychol Med 1998b;28:551-8.

21. Kuerbis AN, Neighbors CJ, Morgenstern J. Depression's Moderation of the Effectiveness of Intensive Case Management With Substance-Dependent Women on Temporary Assistance for Needy Families: Outpatient Substance Use Disorder Treatment Utilization and Outcomes. J Stud Alcohol Drugs 2011;72(2):297-307.

22. Morgenstern J, Blanchard KA, McCrady BS, McVeigh KH, Morgan TJ, Pandina RJ. Effectiveness of Intensive Case Management for Substance-Dependent Women Receiving Temporary Assistance for Needy Families. Am J Pub Health 2006;96(11):2016-23.

\author{
Acknowledgements - Nil \\ Source of Funding - Nil \\ Conflict of Interest - Nil
}

